\title{
Generalized Maxwell Relations in Thermodynamics with Metric Derivatives
}

\author{
José Weberszpil ${ }^{1}$ (D) and Wen Chen ${ }^{2, *}$ \\ 1 Instituto Multidisciplinar-Departamento de Tecnologias e Linguagens, \\ Universidade Federal Rural do Rio de Janeiro, UFRRJ-IM/DTL, Governador Roberto Silveira n/n, \\ Nova Iguaçú, Rio de Janeiro 23890-000, Brazil; josewebe@ufrrj.br or josewebe@gmail.com \\ 2 College of Mechanics and Materials, Hohai University, Jiangning District, Fuchengxi Road 8, \\ Nanjing 211100, China \\ * Correspondence: chenwen@hhu.edu.cn
}

Received: 5 July 2017; Accepted: 27 July 2017; Published: 7 August 2017

\begin{abstract}
In this contribution, we develop the Maxwell generalized thermodynamical relations via the metric derivative model upon the mapping to a continuous fractal space. This study also introduces the total $q$-derivative expressions depending on two variables, to describe nonextensive statistical mechanics and also the $\alpha$-total differentiation with conformable derivatives. Some results in the literature are re-obtained, such as the physical temperature defined by Sumiyoshi Abe.
\end{abstract}

Keywords: deformed maxwell relations; metric derivatives; fractal continuum; generalized statistical mechanics; Legendre structure

\section{Introduction}

Maxwell relations are thermodynamic equations which establish the relations between various thermodynamic quantities (e.g., pressure, $P$, volume, $V$, Entropy, $S$, and temperature, $T$ ) in equilibrium thermodynamics via other fundamental quantities known as thermodynamical potentials- the most important being internal energy, $U$, Helmholtz free energy, $F$, enthalpy, $H$, and Gibbs free energy, $G$.

A Legendre transform converts from a function of one set of variables to another function of a conjugate set of variables. With the use of Legendre transforms, we can study different equations of state. For example, with the adequate change in variables, $U(S, V)$ transforms to $F=F(V, T)$.

In this study, we pursue the development of Maxwell's generalized thermodynamic relations, considering complex systems in which the thermodynamic quantities are expressed by partial metric derivatives (MDs) [1,2]. In order to fulfill this task, we start with the redefinition of the mathematical concept of the derivatives of functions under the physical basis of mappings to a continuous fractal space as proposed in References [3-5]. According to Reference [6], the generalized equations of state (called "fractal" equations of state) are derived on the basis of the experimentally measured thermodynamic variables $P, V$, and $T$, which achieve good agreement with experimental data for argon gas.

To pursue a mathematical basis for physics on fractals, differential vector calculus in a three-dimensional continuum with fractal metric were studied in Reference [5], where the mapping of physical problems on nowhere differentiable fractals into the corresponding problems for a continuum, with a proper fractal metric, is discussed. The authors also showed that there exists an isomorphism of algebras and present a mathematical formalism to differential vector calculus in a continuum with the induced fractal metric. In Reference [7], while studying anomalous diffusion of fluid momentum and Darcy-like law for laminar flow in media with fractal porosity, the authors showed that in the context studied, there exists a homomorphism in certain cases of dimensional parameters. The physical problems on a fractal $\left(F \subset E^{3}\right)$ can be mapped into problems in the $v$-dimensional space $F^{v} \subseteq E^{3}$ 
equipped with the metric induced by the fractal topology, while $v$ is the number of effective degrees of freedom of a random walker on the fractal. The mapping implies a change from the Cartesian coordinates in the embedding Euclidean space to the fractal coordinates in the fractional dimensional space allied to the fractal. Furthermore, the mapping $F \subset E^{3} \rightarrow F^{v} \subseteq E^{3}$ implies the use of metric partial derivatives instead of conventional partial derivatives. So, we can claim that the mapping to a continuous fractal space [3-5] leads naturally to the necessity of modifications in the definition of derivatives. Consequently, the use of the MD $[1,2]$ is justified. The modification of the derivatives in terms of the metric implies a change in the algebra involved, which in turn gives rise to a generalized statistical mechanics with certain generalized definition of entropy, such as in nonextensive statistical mechanics. The use of these deformed-operators can be justified on our proposition that there exists an intimate relation between dissipation, coarse-grained media, and the limit scale of energy among the interactions. It seems that a suitable way to describe the dynamics of complex systems requires the use of the MD and the associated operators instead of the popular fractional derivatives (FD). The $q$-deformed derivative and the correspondent $q$-deformed algebras consequently appear in the context of Tsallis' statistical mechanics and other deformed operators like the Hausdorff and the conformable derivatives.

In considering the appearance of the concept of general multiscale metric derivatives, we can cast the interesting work in Reference [8] (reviewed in Reference [9]) with the effort to build up geometry and field theory in multi-fractional space-time, but there with the name of $q$-derivative. In [9], the author shows, among a vast number of other things, that the multifractal paradigm not only makes the "fractal space-time" idea systematic, but it also provides an explicit form for the dynamics (questions 31 and 40 in the reference). Moreover, the author works with a mapping between the fractional and the integer picture that includes the deformed derivative involved there.

All of the above-referred approaches indicate the systematic attempts to build up mappings that lead to the redefinition of derivatives as structural deformed operators that can be used to describe the dynamics of complex and open systems in the most diverse research lines.

Following this path, in a recent work it was demonstrated that there is a possible relation between $q$-deformed algebras in the two different contexts of statistical mechanics (namely, the Tsallis' framework and the Kaniadakis' scenario), with a local form of a deformed-derivative operator for fractal media-the so-called Hausdorff derivatives-mapped into a continuous medium with a fractal measure, considering the relevant space-time/phase space as fractal or multifractal [1]. One of the authors shows that this approach could be capable of describing dissipative systems, where the traditional Lagrangian formalism fails [2]. Instead, a method of dealing with a mass-dependent Lagrangian was presented.

Following this line of thought, in a recent article [10] it was claimed that the use of deformed operators could be justified based on our proposition that there exists an intimate relation between dissipation, coarse-grained media, and a limit energy scale for the interactions. Concepts and connections like open systems, quasi-particles, energy scale, and the change in the geometry of space-time at its topological level, nonconservative systems, and noninteger dimensions of space-time connected to a coarse-grained medium have been discussed. With this perspective, we argued that deformed (or metric or structural derivatives) — similar to the fractional calculus (FC) — could allow us to describe and emulate certain dynamics without explicit many-body, dissipation, or geometrical terms in the dynamical governing equations. Additionally, we emphasized that the paradigm we adopted was different from the standard approach in the generalized statistical mechanics context [11-13], where the modification in the definition of entropy leads to the modification of the algebra-and consequently, the concept of a derivative [1,2]. This was set up by a mapping into a continuous fractal space [3-5], which naturally yields the need for modifications in the derivatives which we have named deformed, or better, metric derivatives [1,2]. In connection with the metric, the modifications of the derivatives yields a change in the algebra involved, which in turn may lead to a generalized statistical mechanics with some adequate definition of entropy. 
According to Reference [14], there is a relation between entropy and the fractal dimension; the latter can be considered a statistical index, measuring the complexity of a given pattern embedded in the topological space and characterizing a measure of the space-filling capacity. It is possible to define the statistical complexity by using different distance measures [15]. Thus, we claim that with the physical definition of the MD, the complexity can be inherently considered by the generalized thermodynamics characterized in entropic expressions.

To establish a solid basis for the MD and its applications, some experimental tests can be indicated. For instance, the so called fractal differential equations [16]—particular forms of the metric differential equations-were applied to predict the surface temperature of air-cooled cylindrical Li-ion cells. The authors [16] also argue that it can be used to model a variety of non-integer power law scaling phenomena such as turbulence, fractional quantum mechanics, anomalous diffusion, and they elaborate an interesting argument that fractal time models can describe systems with temporal behavior that present strong intermittence and, self-similarity, without a characteristic time scale. Under fractal time average, the time of an event must be infinite instead of the usual finite for systems that possess time scale. Such an example relates the time in which a fluid particle spends in a given vortex. The lack of an average time induces fractional exponents. For details, see references therein.

In numerical contexts, it has been shown that the MD models have distinct advantages over the existing models from the perspective of computational accuracy and efficiency [17].

Here, it seems pertinent to stress the fact that the mathematical tool of MD has advantages when compared to the formalism with the FC (in addition to the numerical ones already observed above). This also includes attempts to formulate non-equilibrium thermodynamics. The Leibniz differentiation rules and the chain rule are preserved for MD, which facilitates the design, development, and research in linear and nonlinear systems, including quantum systems and hydrodynamic models. However, the equivalence between these formalisms needs to be verified in each case. For example, there seems to be some equivalence in the case of water transport in an unsaturated medium [18]. Recall that the formalism of FC is based on non-local operators in its formulation, while that of MD is based on definitions of local operators. However, one claim that non-locality can also be considered with the argument is as follows: we are dealing with open systems and the hereditary effects or the nonlocal effects (memory and spatial effects) are inherent to the fractality, connected to the geometry or the topology of the medium. In an attempt to outline possible connections between formalisms of FC and the MD (the latter being presented in this work), we can indicate Reference [18], where nonlocality is considered with the use of FC. The authors in Reference [18] propose a fractal Richards equation, in which the classical integer-order time derivative of the water content is replaced by a fractal derivative underlying a power law rule in time, whose solution exhibits anomalous non-Boltzmann scaling, attributed to the fractal nature of heterogeneous media. The model was verified by accurately fitting the eight different benchmark experiment data for the water content curves reported in the literature. The wide applicability of this Richards model demonstrates a clear edge of the MD approach over the other existing models.

Additionally, in terms of applications, the authors in Reference [19] show the applicability of what they call fractal models with fractal derivatives to characterize the creep of viscoelastic materials. The applicability of a MD formalism is reinforced by the recent publication in Reference [20], where aspects of Tsallis' entropy in water engineering are studied. Additionally, an effort to unified formalisms can be found in Reference [21].

In this contribution, our focus is on the development of generalized Maxwell relations based on a mapping to a fractal continuum $[4,5,22]$ with the use of MDs. We work with two kinds of MD: the first in the context of generalized nonadditive statistical mechanics called $q$-derivative, and the second called conformable derivatives [23], in the context of fractals and multifractals. All these operators are local metric derivatives. 
In the following section, the reader will see that the concept of physical temperature defined by Abe in Reference [24] emerges naturally in our approach, just like the emergence of the Rényi entropy from the Tsallis entropy and the relation between Sharma-Mittal and Tsallis entropic forms.

The remainder of this paper is outlined as follows: In Section 2, the metric derivatives and mathematical aspects are presented. In Section 3, the deformed Maxwell relations are developed, followed by the Conclusions and Outlook in Section 4 .

\section{Metric Derivatives: Mathematical Aspects}

In this Section, we provide some brief information to review the main forms of the MD. For more details, the readers may see References [1-3,19,25].

\subsection{Conformable Derivative}

This kind of MD was proposed in Reference [23], preserves classical operational properties, and is given by

$$
T_{\alpha} f(t)=\lim _{\epsilon \rightarrow 0} \frac{f\left(t+\epsilon t^{1-\alpha}\right)-f(t)}{\epsilon} .
$$

If the function $f$ is differentiable in a classical sense, the definition above yields

$$
T_{\alpha} f(t)=t^{1-\alpha} \frac{d f(t)}{d t} .
$$

Changing the variable $t \rightarrow 1+\frac{x}{l_{0}}$, one of the authors shows [1] that Equation (2) is nothing but the Hausdorff derivative $[1,3-5,19,25]$ up to a constant, and is valid for differentiable functions.

We can see that the above form of the MD has the same mathematical structure of generic MD for differentiable functions.

Interconnections and equivalence of the metric derivatives was presented in Reference [1] particularly between definitions of fractal derivatives, called Hausdorff derivative in Reference [25], $q$-derivatives in Reference [26], and conformable derivatives in Reference [23]. Therein, a simple scale change in the variable was used to show that for differentiable functions, the conformable derivatives are equivalent to Hausdorff derivatives.

We provide some comments here: The first is that the Maxwell relations that will be stated in Section 3 may involve multiscales, since we are working with different state variables that can be described by different parameters (e.g., different entropic indices). Moreover, we reinforce that all the metric derivatives referred to here are local operators, and for this reason cannot be simply proportional to the Riemann-Liouville derivative in the context of FC.

\subsection{The q-Derivative in a Nonextensive Context}

With the generalized nonadditive $q$-entropy as the main motivation, the $q$-derivative sets up a deformed algebra and considers that the $q$-exponential is an eigenfunction of $D_{(q)}$ [26]. Borges [26] proposed the operator for $q$-derivative as given below:

$$
D_{(q)} f(x) \equiv \lim _{x^{\prime} \rightarrow x} \frac{f\left(x^{\prime}\right)-f(x)}{x^{\prime} \ominus_{q} x}=[1+(1-q) x] \frac{d f(x)}{d x} .
$$

Here, $\ominus_{q}$ is the deformed difference operator,

$$
x^{\prime} \ominus_{q} x \equiv \frac{x^{\prime}-x}{1+(1-q) x} \quad(x \neq 1 /(q-1)) .
$$

If we write $x^{\prime}=x \oplus_{q} \Delta x=x+\triangle x+(1-q) x \triangle x$, we will have $x^{\prime} \ominus_{q} x=\Delta x$, where $\oplus_{q}$ is the deformed sum operator. 
Additionally,

$$
d_{q}(x) \equiv \lim _{x^{\prime} \rightarrow x} x^{\prime} \ominus_{q} x=\frac{d x}{1+(1-q) x} \quad(x \neq 1 /(q-1)) .
$$

In this way, the expression (3) can be also rewritten as in Reference [27],

$$
D_{(q)} f(x) \equiv \lim _{\triangle x \rightarrow 0} \frac{f\left(x \oplus_{q} \triangle x\right)-f(x)}{\triangle x} .
$$

This is the key for the multivariable $q$-Taylor expansion.

Recently, one of the authors has shown [1] that the main parameters of MD, Hausdorff derivative, and the $q$-derivative can be connected by

$$
1-q=\frac{(1-\zeta)}{l_{0}}
$$

where the characteristic scale $l_{0}$ appears in the context of the mappings in References [3-5].

We concluded that the deformed or metric $q$-derivative is the first-order expansion of the Hausdorff derivative, and that there is a strong connection between these formalisms by means of a fractal metric.

An important point to emphasize here concerns the appearance of multifractals, since multifractal measures are formally used to explain systems with scaling properties [28]. In Reference [28], an approach based on generalized Tsallis dimensions is used for the formulation of mutual information related dependence coefficients in the multifractal domain. This is only one example indicating that the concepts of fractals, scales, and multifractals are present in our approach, since $q$-derivatives are one of several possible forms of metric derivatives. In Reference [2], the authors pointed out that the deformation parameter or entropic index, $q$-occupying an important place in the description of those complex systems-describes deviations from standard Lie symmetries, and the formalism of metric derivatives aimed to accommodate scale invariance in a system with multifractal properties to the thermodynamic formalism, and considered the relevant space-time/phase space as fractal or multifractal.

\subsection{The q-Total Differentiation in Nonextensive Context}

For one variable, let us expand $f\left(x^{\prime}\right)=f\left(x \oplus_{q} \triangle x\right)$ around $x$ [27]:

$$
f\left(x^{\prime}\right)=f(x)+\left.\left(x^{\prime}-x\right) \frac{d f\left(x^{\prime}\right)}{d x^{\prime}}\right|_{x^{\prime}=x}+O\left((\triangle x)^{2}\right),
$$

which can be written as

$$
f\left(x \oplus_{q} \triangle x\right)=f(x)+\left.\triangle x[1+(1-q) x] \frac{d f\left(x^{\prime}\right)}{d x^{\prime}}\right|_{x^{\prime}=x}+O\left((\triangle x)^{2}\right),
$$

where $\triangle x \rightarrow 0$.

Now, we can extend to more than one variable with the expansion around $x, y$. Assuming a possible different $q$-parameter for $x, y$ variables, a total differential is:

$$
\begin{aligned}
f\left(x \oplus_{q} \triangle x, y \oplus_{q^{\prime}} \triangle y\right)=f(x, y)+ & \left.\Delta x[1+(1-q) x] \frac{\partial f\left(x^{\prime}\right)}{\partial x^{\prime}}\right|_{x^{\prime}=x, y=c t e} \\
& +\left.\triangle y\left[1+\left(1-q^{\prime}\right) y\right] \frac{\partial f\left(y^{\prime}\right)}{\partial y^{\prime}}\right|_{y^{\prime}=y, x=c t e}+O\left((\triangle x)^{2},(\triangle y)^{2}\right) .
\end{aligned}
$$

In the limit $\triangle x \rightarrow 0, \Delta y \rightarrow 0$, the expression above can be written as 


$$
f\left(x \oplus_{q} d x, y \oplus_{q} d y\right)=f(x, y)+\left.\frac{\partial_{q} f(x, y)}{\partial x}\right|_{y} d x+\left.\frac{\partial_{q^{\prime}} f(x, y)}{\partial y}\right|_{x} d y .
$$

\subsection{The Leibiniz and the Chain Rule Hold for Metric Derivative}

As the reader can readily check, for the MD the Leibniz rule holds $D^{\alpha}(f g)=g D^{\alpha} f+f D^{\alpha} g$; and similarly, for $q$-derivative: $D_{q}(f g)=g D_{q} f+f D_{q} g$. For composed functions, the chain rule holds true as well:

$$
\begin{aligned}
& D^{\alpha}[f \circ g](x)=\frac{d f(g(x))}{d g} D^{\alpha} g(x), \\
& D_{q}[f \circ g](x)=\frac{d f(g(x))}{d g} D_{q} g(x) .
\end{aligned}
$$

\section{Deformed Maxwell Relations}

In this section, we pursue the investigation of a version of the MD to study a possible version of generalized thermodynamics. The intention is to build a tool to study the dynamics of complex systems such as porous media or some viscous systems.

Before proceeding further, in order to clarify some points here, we now make a few brief remarks about entropy. There are some controversies regarding the connections of the concept of entropy in the sense of Clausius and in the context of information theory-that is, with entropy and disorder [29]. For example, in Reference [30], the author uses liquid crystals to show that an increase in the order of the refereed system follows the increase in entropy, which could be considered impossible in the context of the connection between entropy and disorder. Additionally, against the idea that entropy and disorder are always the same, see Reference [31]. In the sense of information theory, entropy is related to aspects and problems of information and communication [32]. In addition, in the context of information theory, the important concept of informational energy is that related to classical mechanics, and can be considered as a measure of the uncertainty or randomness of a probability system [32]. Here, one adopts the conciliatory sense-namely, that entropy describes the amount of disorder or randomness in a system bearing energy or information [32], and that can be also used to evaluate aspects of uncertainty. In this broader sense, there are some generalized entropic forms which are particularly inspired in the context of information theory; for instance, Havdra-Charvat-Tsallis (HCT), Sharma-Mittal (SM), Sharma-Taneja (ST), and attempts to construct a generalized statistics with these forms. Examples of these attempts are based on the entropy concept like Rényi, HCT, Kaniadakis, and incomplete information-based theory [33,34].

Now, consider a mapping to a continuous fractal space [3-5] which leads naturally to the necessity of modifications in the derivatives, called the MD [1,2]. In the new continuous space, we call $S_{q}$, $S_{\alpha}$ metric entropies, depending on the metric considered.

\section{1. q-Deformed Maxwell Relations}

Consider $U_{q}$ as some internal energy that is a function of the entropy $S_{q}$ and volume $V_{q^{\prime}}$ : $U_{q}=U_{q}\left(S_{q}, V_{q^{\prime}}\right)$, where the entropic indices $q$ and $q^{\prime}$ are not necessarily the same.

The total $q$-derivative can be written as

$$
\begin{gathered}
U_{q}\left(S_{q} \oplus_{q} \Delta S_{q}, V_{q^{\prime}} \oplus_{q^{\prime}} \Delta V_{q^{\prime}}\right)-U_{q}\left(S_{q}, V_{q^{\prime}}\right) \\
=U_{q}\left(S_{q} \oplus_{q} \Delta S_{q}, V \oplus_{q^{\prime}} \Delta V_{q^{\prime}}\right)-U_{q}\left(S_{q}, V \oplus_{q^{\prime}} \Delta V_{q^{\prime}}\right) \\
+U_{q}\left(S_{q}, V \oplus_{q^{\prime}} \Delta V_{q^{\prime}}\right)-U_{q}\left(S_{q}, V_{q^{\prime}}\right) .
\end{gathered}
$$


In the limit of $\Delta V_{q^{\prime}}, \nabla S_{q} \rightarrow 0$, we find for the total $q$-derivative of $U_{q}$

$$
\begin{aligned}
D_{q} U_{q} & =\left.\left[1+(1-q) S_{q}\right] \frac{\partial U_{q}}{\partial S_{q}}\right|_{V_{q^{\prime}}} d S_{q}+\left.\left[1+\left(1-q^{\prime}\right) V_{q^{\prime}}\right] \frac{\partial U_{q}}{\partial V_{q^{\prime}}}\right|_{S_{q}} d V_{q^{\prime}} \\
& =\left.\frac{\partial_{q} U_{q}}{\partial S_{q}}\right|_{V} d S_{q}+\left.\frac{\partial_{q^{\prime}} U_{q^{\prime}}}{\partial V_{q^{\prime}}}\right|_{S} d V_{q^{\prime}} .
\end{aligned}
$$

Now, in the same way, consider $U_{q}=U_{q}\left(T_{q}, S_{q}\right)$, where the variable $T_{q}$ is introduced and will be made clear in what follows.

The total $q$-derivative can be written as:

$$
D_{q} U_{q}\left(T_{q}, S_{q}\right)=\left.\frac{\partial_{q} U_{q}}{\partial T_{q}}\right|_{S_{q}} d T_{q}+\left.\frac{\partial_{q} U_{q}}{\partial S_{q}}\right|_{T_{q}} d T_{q}
$$

Here, $T_{q}=\left.\frac{\partial U_{q}}{\partial S_{q}}\right|_{q_{q^{\prime}}}$ is not a temperature. It is similar to $k^{T} \beta^{-1}$ in the nonextensive formalisms, and is sometimes related to the Lagrange multiplier in the maximum entropy variational approach.

\subsubsection{Legendre Transform and Helmholtz Free Energy, Enthalpy, and Gibbs Free Energy}

Suppose a transform with change of variables in such a way that $U_{q}\left(S_{q}, V_{q}\right)$ transfers to $F_{q}=F_{q}\left(V_{q}, T_{q}\right)$, with $F_{q}=U_{q}-T_{q} S_{q}$ being the Helmholtz free energy.

Adding and subtracting Equation (16) to Equation (15) and considering that $F_{q}$ only depends on $\left(V_{q}, T_{q}\right)$, yields

$$
\begin{gathered}
D_{q}\left(U_{q}-T_{q} S_{q}\right)=D_{q} F_{q}=\left.\frac{\partial_{q} U_{q}}{\partial V_{q}}\right|_{S} d V_{q}-\left.\frac{\partial_{q}\left(T_{q} S_{q}\right)}{\partial T_{q}}\right|_{S} d T_{q}, \\
\left.\frac{\partial_{q} U_{q}}{\partial S_{q}}\right|_{V_{q}}=T_{q}\left[1+(1-q) S_{q}\right] .
\end{gathered}
$$

The expression in Equation (18) is nothing else than the physical temperature defined by Abe in Reference [24].

Now consider the partial $q$-derivatives of $T_{q} S_{q}$ and of $P_{q} V_{q}$

$$
\begin{aligned}
& \left.\frac{\partial_{q}\left(P_{q} V_{q^{\prime}}\right)}{\partial P_{q}}\right|_{V}=V_{q^{\prime}}\left[1+\left(1-q^{\prime}\right) P_{q^{\prime}}\right], \\
& \left.\frac{\partial_{q}\left(P_{q} V_{q}\right)}{\partial V_{q}}\right|_{P}=P_{q^{\prime}}\left[1+\left(1-q^{\prime}\right) V_{q^{\prime}}\right] .
\end{aligned}
$$

Analogously to $U_{q}$, consider the enthalpy function given by $H_{q}=U_{q}+P_{q} V=H_{q}\left(S_{q}, P_{q^{\prime}}\right)$. The total $q$-derivative of $H_{q}$ can be written as

$$
\begin{aligned}
D_{q}\left(U_{q}+P_{q} V\right) & =D_{q} H_{q}=\left.\frac{\partial_{q} U_{q}}{\partial S_{q}}\right|_{V_{q}} d S+\left.\frac{\partial_{q}\left(P_{q} V_{q}\right)}{\partial P_{q}}\right|_{V_{q}} d P_{q} \\
\left.\frac{\partial_{q} U_{q}}{\partial V}\right|_{S_{q}} & =-P_{q}[1+(1-q) V] .
\end{aligned}
$$

Finally, the $q$-deformed differential forms of $U_{q}, F_{q}, H_{q}$ are given by

$$
\begin{aligned}
D_{q} U_{q} & =T_{q}\left[1+(1-q) S_{q}\right] d S_{q}-P_{q}\left[1+(1-q) V_{q}\right] d V_{q}, \\
D_{q} F_{q} & =-P_{q}\left[1+(1-q) V_{q}\right]-S_{q}\left[1+(1-q) T_{q}\right] d T_{q}, \\
D_{q} H_{q} & =T_{q}\left[1+(1-q) S_{q}\right] d S_{q}+V_{q}\left[1+(1-q) P_{q}\right] d P_{q} .
\end{aligned}
$$


In a similar way, for the Gibbs free energy $G_{q}$, we have

$$
D_{q} G=-S_{q}\left[1+(1-q) T_{q}\right] d T_{q}+V_{q}\left[1+(1-q) P_{q}\right] d P_{q} .
$$

With the help of the above equations, we can rewrite Equation (15) as

$$
D_{q} U_{q}=T_{P h y s} d S_{q}-P_{P h y s} d V_{q} .
$$

\subsubsection{The $q$-Deformed Maxwell Relations}

Below we collect all the Maxwell relations obtained by the present approach with $q$-derivatives. They are collected in Table 1.

Table 1. $q$-Deformed Maxwell relations.

\begin{tabular}{lc}
\hline$\left.\frac{\partial_{q} U_{q}}{\partial V_{q}}\right|_{S_{q}}=-P_{q}\left[1+(1-q) V_{q}\right]$, & $\left.\frac{\partial_{q} U_{q}}{\partial S_{q}}\right|_{V_{q}}=T_{q}\left[1+(1-q) S_{q}\right]$, \\
\hline$\left.\frac{\partial_{q} F_{q}}{\partial V_{q}}\right|_{T_{q}}=-P_{q}\left[1+(1-q) V_{q}\right]$, & $\left.\frac{\partial_{q} F_{q}}{\partial T_{q}}\right|_{V_{q}}=-S_{q}\left[1+(1-q) T_{q}\right]$, \\
\hline$\left.\frac{\partial_{q} H_{q}}{\partial P_{q}}\right|_{S_{q}}=V_{q}\left[1+(1-q) P_{q}\right]$, & $\left.\frac{\partial_{q} H_{q}}{\partial S_{q}}\right|_{P_{q}}=T_{q}\left[1+(1-q) S_{q}\right]$, \\
\hline$\left.\frac{\partial_{q} G_{q}}{\partial P_{q}}\right|_{T_{q}}=-V\left[1+(1-q) P_{q}\right]$, & $\left.\frac{\partial_{q} G_{q}}{\partial T_{q}}\right|_{P_{q}}=-S_{q}\left[1+(1-q) T_{q}\right]$, \\
\hline
\end{tabular}

One important point that can be emphasized is the difference between the entropic indices in the Maxwell relations. Those differences could indicate that there are additional ways to interpret the possible existence of the so-called $q$-triplet [35]. A large updated bibliography can be found in Reference [36] pursuing the task of contextualizing the entropic index as something beyond a simple parameter of data adjustment, and that has been researched and justified. Even though it is not one of the objectives of this article, we suggest that the entropic index is related to the metric, which in turn is related to the existing interactions. So, we call the reader's attention to the possible relation between the entropic index and the metric, justifying the use of the appropriate metric derivative to each physical phenomenon.

\subsubsection{Emergence of Rényi Entropy from $S_{q}$ as Tsallis Entropy} led to

Consider that from Equation (27) $d Q_{q}=T_{P h y s} d S_{q}$ as the inexact deformed heat transfer, we are

$$
d S_{q}=\frac{d Q_{q}}{T_{P h y s}},
$$

or

$$
(d S)_{q}=\frac{d Q_{q}}{T_{q}} \times \frac{1}{\left[1+(1-q) S_{q}\right]}=\frac{d s_{q}}{\left[1+(1-q) S_{q}\right]} .
$$

Note that the above expression is consistent with the differentiation in the fractal continuous space given by [26]

$$
\left(d_{q} x\right)=\frac{d x_{q}}{\left[1+(1-q) x_{q}\right]} .
$$

A direct integration leads to

$$
(\triangle S)_{q}^{R}=\frac{1}{1-q} \ln \left[1+(1-q) S_{q}^{T}\right] .
$$

That is the relation between Rényi-Tsallis entropies which is also given in Reference [24] and can indicate an emergence of micro to macro properties.

Rényi introduced a family of entropy functionals called the alpha entropies by using the Kolmogorov-Nagumo averages [37]. According to Reference [37], the role of scale translation would 
be studied in a systematic manner, since the procedure of estimating parameters is sensitive to the scale on which the experimental data are presented.

The Rényi entropy is additive, while the Tsallis entropy is not. This means that the thermodynamic macroscopic additivity for the entropy emerges even from a starting point for entropy based on nonadditivity. There is a nonlinear scale for the connection of the Renyi-Tsallis entropies, and this could indicate the possible emergence of additivity. This can be a consequence of complexity, in the sense that there are repeating patterns to form new complex patterns.

Here, the entropic form $S_{q}$ can be seen as metric entropy from a microscopic view. On the other hand, considering the standard second law in the Clausius form, $(\triangle S)_{q}^{R}$ leads to the validity of the second law for the metric entropy $S_{q}$ :

$$
\frac{\mathrm{d} Q_{q}}{T_{q}} \geq 0
$$

That is, $d S_{q} \geq 0$.

\subsection{Conformable Deformed Maxwell Relations}

In order to build up the Maxwell relations with the conformable derivatives, we expand a given function $f$ with an equivalent form of Taylor expansion using integer polynomials to the first order [38], instead of noninteger [39] order polynomial. This merely reflects the fact that the space of concerned functions is considered differentiable.

The $\alpha$-total differentiation of a differentiable function can be developed by the infinitesimal difference:

$$
d_{\alpha} f \equiv f\left(x+\varepsilon_{x} x^{1-\alpha}, y+x+\varepsilon_{x} x^{1-\alpha} \varepsilon_{y} y^{1-\alpha}\right)-f(x, y),
$$

which can be written in terms of conformable partial derivatives as

$$
\begin{aligned}
d_{\alpha} f & =f\left(x+\varepsilon_{x} x^{1-\alpha}, y+x+\varepsilon_{x} x^{1-\alpha} \varepsilon_{y} y^{1-\alpha}\right)-f(x, y) \\
& =f\left(x+\varepsilon_{x} x^{1-\alpha}, y+\varepsilon_{y} y^{1-\alpha}\right)-f\left(x+\varepsilon_{x} x^{1-\alpha}, y\right)+f\left(x+\varepsilon_{x} x^{1-\alpha}, y\right)-f(x, y) \\
& =\frac{\partial_{\alpha} f}{\partial x} \varepsilon_{x}+\frac{\partial_{\alpha} f}{\partial y} \varepsilon_{y}=\frac{\partial_{\alpha} f}{\partial x}(d x)+\frac{\partial_{\alpha} f}{\partial y}(d y) .
\end{aligned}
$$

Next, following the analogous approach of $q$-derivative to obtain Legendre Transform and Helmholtz free energy, enthalpy, and Gibbs free energy, we can obtain the $\alpha$-deformed Maxwell relations that we collect in Table 2.

Table 2. $\alpha$-Deformed Maxwell relations.

\begin{tabular}{cc}
\hline$\left.\frac{\partial_{\alpha} U_{\alpha}}{\partial V}\right|_{S_{\alpha}}=-P_{\alpha} V^{1-\alpha}$, & $\left.\frac{\partial_{\alpha} U_{\alpha}}{\partial S_{\alpha}}\right|_{V}=T_{\alpha} S_{\alpha}^{1-\alpha}$, \\
\hline$\left.\frac{\partial_{\alpha} F_{\alpha}}{\partial V}\right|_{T_{\alpha}}=-P_{\alpha} V^{1-\alpha}$, & $\left.\frac{\partial_{\alpha} F_{\alpha}}{\partial T_{\alpha}}\right|_{V}=-S_{\alpha} T_{\alpha}^{1-\alpha}$, \\
\hline$\left.\frac{\partial_{\alpha} H_{\alpha}}{\partial P_{\alpha}}\right|_{S_{\alpha}}=V P_{\alpha}^{1-\alpha}$, & $\left.\frac{\partial_{\alpha} H_{\alpha}}{\partial S_{\alpha}}\right|_{P_{\alpha}}=T_{\alpha} S_{\alpha}^{1-\alpha}$, \\
\hline$\left.\frac{\partial_{\alpha} G_{\alpha}}{\partial P_{\alpha}}\right|_{T_{\alpha}}=-V P_{\alpha}^{1-\alpha}$, & $\left.\frac{\partial_{\alpha} G_{\alpha}}{\partial T_{\alpha}}\right|_{P_{\alpha}}=-S_{\alpha} T_{\alpha}^{1-\alpha}$, \\
\hline
\end{tabular}

This is almost identical to the results in Reference [6], except for the gamma function pre factor. Here $\alpha$ is different from that work as well. All procedures to obtain a fractal equation of state are simpler than in Reference [6], and can be done to compare with experimental data. A further investigation along this line will be presented in a future work.

In each of the above Maxwell relations, the $\alpha$-parameter does not necessarily need to be the same for all Maxwell relations. In this study, we maintain the same in order to compare to results in Reference [6].

Note that in Reference [6], the parameter $\alpha$ is very close to 1 . This is important to for the approximation there to be valid; that is, only lower order for $i$ parameter $(i=0)$ in the expansion 
$A 3, A 4$ are kept. This in fact makes the procedure used there local. Apparently, the use of complicated fractional calculus seems unnecessary compared to the conformable or the $q$-deformed MD procedures.

Considering $d U=T_{\text {Phys }} d S_{\alpha}$, we can write

$$
d S_{\alpha}=\frac{d Q}{T p h y S}=\frac{d Q}{T} \times \frac{1}{S^{1-\alpha}}=\frac{d S}{S^{1-\alpha}},
$$

which is consistent with the Improper Riemann integral [23]:

$$
I_{a}^{\alpha}(f)(t)=\int_{a}^{t} f(x) d^{\alpha} x=\int_{a}^{t} f(x) x^{\alpha-1} d x,
$$

where $d x^{\alpha}=\frac{1}{x^{1-\alpha}} d x$. Integrating Equation (35) will give

$$
(\triangle S)_{\alpha}=\frac{S^{\alpha}}{\alpha}
$$

Changing the variable $S \rightarrow 1+\frac{S}{l_{0}}$ in Equation (35), we obtain, after integration, the result $l_{0}\left(1+\frac{S}{l_{0}}\right)^{\alpha}$, which is related to the Hausdorff derivative up to a constant and is valid for differentiable functions [2]. Consequently, we have

$$
(\triangle S)_{\alpha}=\frac{l_{0}\left(1+\frac{S}{l_{0}}\right)^{\alpha}}{\alpha} .
$$

If we consider $\alpha=\frac{1-r}{1-q}$ and $l_{0}=\frac{l_{0}^{\prime}}{1-q}$, the result is

$$
(\triangle S)_{\alpha}=\frac{l_{0}^{\prime}\left(1+(1-q) \frac{S}{l_{0}^{\prime}}\right)^{\frac{1-r}{1-q}}}{1-r} .
$$

For $l_{0}^{\prime}=1$, this is related to the Sharma-Mittal (SM) and Tsallis entropic forms

$$
(\triangle S)_{\alpha}=S_{q, r}^{S M}+\frac{1}{1-r}=\frac{\left[1+(1-q) S_{q}^{T}\right]^{\frac{1-r}{1-q}}}{1-r} .
$$

It is well-known that for $q=r$, the Tsallis entropy is the result. For the limit $r \rightarrow 1, q \neq 1$, the Rényi entropy emerges. For $r \rightarrow 1, q \rightarrow 1$, the Boltzmann-Gibbs is the limiting entropic form. We conclude that the SM entropy is a kind of re-parametrized geometric average of the Tsallis entropy, which is on its own an exponentiation of Rényi entropic form. The Rényi entropy is a smoothing of both the Tsallis and the Sharma-Mittal entropic forms.

\section{Conclusions and Outlook}

All foregoing presented results give strong evidence to support the paradigm of the MD in the attempt to generalize the thermodynamics. In short, we summarize our findings by the following remarks:

Considering a mapping to the continuous fractal space, we have obtained the generalized Maxwell relations with the use of the MD such as the $q$-derivative in the nonextensive context and the conformable derivative.

To obtain the $q$-deformed and $\alpha$-deformed Maxwell relations, the $q$-total derivative and the $\alpha$-total derivative were developed. The physical temperature, $T_{P h y s}$, in Reference [24] appears naturally in our formalism.

The emergence of the Rényi entropy in the nonextensive context and the Sharma-Mittal-like entropy is obvious. 
For the cases studied, the Maxwell relations of classical thermodynamics are recovered for $\alpha \rightarrow 1$, $q \rightarrow 1$.

In future works, we intend to study the applications of the MD toward the development of a generalized thermodynamics on a solid physical basis within fractal, multifractal under complex systems context.

Additionally, as an outlook for possible research, Reference [40] and some approximate approaches in References [41-43], there are indications that the use of MD and variational principles, that perspectives of applications seem to open for modeling quantum systems, including the representation of quantum mechanics by hydrodynamic models, including those with stochastic processes [44].

Acknowledgments: One of the authors was supported by the National Natural Science Foundation of China (Nos. 11572111, 11372097), the 111 project (Grant no. B12032). Thanks to J. A. Helayël-Neto for fruitfull discussions and for reading the manuscript.

Author Contributions: Both authors José Weberszpil and Wen Chen contributed equally to the article and its writing.

Conflicts of Interest: The authors declare no conflict of interest.

\section{References}

1. Weberszpil, J.; Lazo, M.J.; Helayël-Neto, J.A. On a connection between a class of image-deformed algebras and the Hausdorff derivative in a medium with fractal. Physica A 2015, 436, 399-404.

2. Weberszpil, J.; Helayël-Neto, J.A. Variational Approach and Deformed Derivatives. Physica A 2016, 450, $217-227$.

3. Balankin, A.S.; Elizarraraz, B.E. Map of fluid flow in fractal porous medium into fractal continuum flow. Phys. Rev. E 2012, 85, 056314.

4. Balankin, A.S.; Elizarraraz, B.E. Hydrodynamics of fractal continuum flow. Phys. Rev. E 2012, 85, 025302.

5. Balankin, A.S.; Bory-Reyes, J.; Shapiro, M. Towards a physics on fractals: Differential vector calculus in three-dimensional continuum with fractal metric. Physica A 2016, 444, 345-359.

6. Meilanov, R.P.; Magomedov, R.A. Thermodynamics in Fractional Calculus. J. Eng. Phys. Thermophys. 2014, 87, 1521-1531.

7. Balankin, A.S.; Valdivia, J.C.; Marquez, J.; Susarrey, O.; Solorio-Avila, M.A. Anomalous diffusion of fluid momentum and Darcy-like law for laminar flow in media with fractal porosity. Phys. Lett. A 2016, 380, 2767-2773.

8. Calcagni, G. Geometry and field theory in multi-fractional spacetime. J. High Energy Phys. 2012, 2012, 1-75.

9. Calcagni, G. Multifractional theories: An unconventional review. J. High Energy Phys. 2017, 2017, 1-109.

10. Weberszpil J.; Helyël-Neto, J.A. Structural scale $q$-derivative and the LLG equation in a scenario with fractionality. EPL 2017, 117, doi:10.1209/0295-5075/117/50006.

11. Tsallis, C. Possible generalization of Boltzmann-Gibbs statistics. J. Stat. Phys. 1988, 52, 479-487.

12. Tsallis, C. Nonadditive entropy and nonextensive statistical mechanics-An overview after 20 years. Braz. J. Phys. 2009, 39, 337-356.

13. Tsallis, C. Introduction to Nonextensive Statistical Mechanics-Approaching a Complex World; Springer: New York, NY, USA, 2009; pp. 1-382.

14. Sparavigna, A.C. Entropies and fractal dimensions. Philica 2016, 2016, 1-5.

15. Radhakrishnan, C.; Chinnarasu, R.; Jambulingam, S. A Fractional Entropy in Fractal Phase Space: Properties and Characterization. Int. J. Stat. Mech. 2014, 2014, 1-16.

16. Reyes-Marambio, J.; Moser, F.; Gana, F.; Severino, B.; Calderón-Muñoz, W.R.; Palma-Behnke, R.; Estevez, P.A.; Orchord, M.; Cortés, M. A fractal time thermal model for predicting the surface temperature of aircooled cylindrical Li-ion cell based on experimental measurements. J. Power Sources 2016, 306, 636-645.

17. Liang, Y.; Allen, Q.Y.; Chen, W.; Gatto, R.G.; Colon-Perez, L.; Mareci, T.H.; Magin, R.L. A fractal derivative model for the characterization of anomalous diffusion in magnetic resonance imaging. Commun. Nonlinear Sci. Numer. Simul. 2016, 39, 529-537.

18. Sun, H.G.; Meerschaert, M.M.; Zhang, Y.; Zhu, J.; Chen, W. A fractal Richards' equation to capture the non-Boltzmann scaling of water transport in unsaturated media. Adv. Water Resour. 2013, 52, 292-295. 
19. Cai, W.; Chen, W.; Xu, W. Characterizing the creep of viscoelastic materials by fractal derivative models. Int. J. Non-Linear Mech. 2016, 87, 58-63.

20. Singh, V.P. Introduction to Tsallis Entropy Theory in Water Engineering; CRC Press, Taylor \& Francis Group, LLC: New York, NY, USA, 2016, ISBN:9781138747944-CAT\# K32605.

21. Chen, W.; Liang, Y. New methodologies in fractional and fractal derivatives modeling. Chaos Solitons Fractals 2017, 102, 72-77.

22. Bashkirov, A.G. Comment on "Stability of Tsallis entropy and instabilities of Rényi and normalized Tsallis entropies: A basis for q-exponential distributions". Phys. Rev. E 2005, 72, 028101.

23. Khalil, R.; Al Horani, M.; Yousef, A.; Sababheh, M. A new Denition of Fractional Derivative. J. Comput. Appl. Math. 2014, 264, 65-70.

24. Abe, S.; Martınez, S.; Pennini, F.; Plastino, A. Nonextensive thermodynamic relations. Phys. Lett. A 2001, 281, 126-130.

25. Chen, W. Time-space fabric underlying anomalous diffusion. Chaos Solitons Fractals 2006, 28, $923-929$.

26. Borges, E.P. A possible deformed algebra and calculus inspired in nonextensive thermostatistics. Physica A 2004, 340, 95-101.

27. Costa, B.G. Aplicação de Uma Estrutura Algébrica não Aditiva em Teoria Quântica. Ph.D. Thesis, Universidade Federal da Bahia-UFBA, Salvador, Brazil, 2015; 267p.

28. Angulo, J.M.; Esquivel, F.J. Multifractal Dimensional Dependence Assessment Based on Tsallis Mutual Information. Entropy 2015, 17, 5382-5401.

29. Wright, P.G. Entropy and Disorder. Contemp. Phys. 1970, 11, 581-588.

30. Styer, D.F. Insight into entropy. Am. J. Phys. 2000, 68, 1090-1096.

31. Lambert, F.L. Disorder-A Cracked Crutch for Supporting Entropy Discussions. J. Chem. Educ. 2002, 79, 187, doi:10.1021/ed079p187.

32. Calin, O.; Udrişte, C. Geometric Modeling in Probability and Statistics; Springer International Publishing: Cham, Switzerland, 2014, ISBN:978-3-319-07778-9.

33. Wang Q.A. Probability distribution and entropy as a measure of uncertainty. J. Phys. A Math. Theor. 2005, 41, 065004.

34. Wang, Q.A. Incomplete statistics-nonextensive generalizations of statistical mechanics. Chaos Solitons Fractals 2001, 12, 1431-1437.

35. Tsallis, C. Generalization of the possible algebraic basis of $q$-triplets. Eur. Phys. J. Spec. Top. 2017, 226, 455-466.

36. Nonextensive Statistical Mechanics and Thermodynamics: Bibliography *. Available online: http://tsallis. cat.cbpf.br/TEMUCO.pdf (accessed on 7 August 2017).

37. Naudts, J. Generalised Thermostatistics; Springer-Verlag London Limited: London, UK, 2011; p. 132, ISSN: 978-0-85729-355-8.

38. Abdeljawad, T. On conformable fractional calculus. J. Comput. Appl. Math. 2015, 279, 57-66.

39. Atangana, A.; Baleanu, D.; Alsaedi, A. New properties of conformable derivative. Open Math. 2015, 13, 889-898.

40. Weberszpil, J.; Helayël-Neto, J.A. Extension and Applications of a Variational Approach with Deformed Derivatives. arXiv 2017, arXiv:1706.09504.

41. Weberszpil, J.; Helayël-Neto, J.A. On the Zitterbewegung Transient Regime in a Coarse-Grained Space-Time. J. Adv. Phys. 2015, 7, 2347-3487.

42. Weberszpil, J.; Helayël-Neto, J.A. Anomalous g-Factors for Charged Leptons in a Fractional Coarse-Grained Approach. Adv. High Energy Phys. 2014, doi:10.1155/2014/572180.

43. Weberszpil, J.; Godinho, C.F.L.; Cherman, A.; Helayël-Neto, J.A. Aspects of the Coarse-Grained-Based Approach to a Low-Relativistic Fractional Schrödinger Equation. In Proceedings of the 7th Conference Mathematical Methods in Physics-ICMP 2012, Trieste, Italy, 16-20 April 2012; pp. 1-19.

44. Kodama, T.; Koide, T. Variational Principle of Hydrodynamics and Quantization by Stochastic Process. arXiv 2014, arXiv:1412.6472.

(C) 2017 by the authors. Licensee MDPI, Basel, Switzerland. This article is an open access article distributed under the terms and conditions of the Creative Commons Attribution (CC BY) license (http:// creativecommons.org/licenses/by/4.0/). 